\title{
The Value of Day-Ahead Solar Power Forecasting Improvement
}

\author{
Carlo Brancucci Martinez-Anido ${ }^{1}$, Benjamin Botor $^{1}$, Anthony R. Florita ${ }^{1}$, Caroline Draxl ${ }^{1}$, \\ Siyuan $\mathrm{Lu}^{2}$, Hendrik F. Hamann ${ }^{2}$, Bri-Mathias Hodge ${ }^{1 *}$ \\ ${ }^{1}$ National Renewable Energy Laboratory, Golden, CO 80401, USA \\ ${ }^{2}$ IBM TJ Watson Research Center, Yorktown Heights, NY 10598, USA
}

\begin{abstract}
The value of day-ahead solar power forecasting improvements was analyzed by simulating the operation of the Independent System Operator - New England (ISO-NE) power system under a range of scenarios with varying solar power penetrations and solar power forecasting improvements. The results showed how the integration of solar power decreased operational electricity generation costs, by decreasing fuel and variable operation and maintenance costs, while decreasing start and shutdown costs of fossil fueled conventional generators. Solar power forecasting improvements changed the impacts that the uncertainty of solar power has on bulk power system operations; electricity generation from the fast start and lower efficiency power plants, ramping of all generators, start and shutdown costs, and solar power curtailment were all reduced. These impacts led to a reduction in overall operational electricity generation costs in the system that translates into an annual economic value for improving solar power forecasting.
\end{abstract}

\section{Introduction}

The decrease in the cost of photovoltaic (PV) panels and associated technology in recent years [1], together with State Renewable Portfolio Standards have contributed to increased solar power investment in the United States [2]. The resulting integration of increasing penetrations of solar power into power systems has challenged utilities and power system operators alike, and will continue to do so with increasing penetration rates. The variability and uncertainty characteristics of solar power represent major hurdles from an operational perspective. Furthermore, the zero marginal cost of solar power impacts the electric grid from the economic and market viewpoints. There is a growing need for solar power forecasting, especially for power systems in which solar power represents a significant share of the electricity generation mix. Several balancing authorities already use solar power forecasting technologies in their daily operations, including the California Independent System Operator (CAISO), the Sacramento Municipal Utility District (SMUD), Southern California Edison (SCE), and Xcel Energy [3].

The integration of solar power in a power system has been a very common research topic during the past decade. Some present or expected future large solar power penetrations around the world have raised several solar integration issues that were not relevant at lower penetration levels. For instance, the "duck curve" is an effect of large instantaneous solar power penetrations in which solar power generation corresponds to a large portion of electricity demand during the middle of

\footnotetext{
${ }^{*}$ Corresponding author. Tel: +1-303-384-6981

E-mail address: bri.mathias.hodge@nrel.gov (Bri-Mathias Hodge)

Page | 1
}

(C) 2016. This manuscript version is made available under the Elsevier user license 
the day. One challenge that this effect causes to power system operations is the ability of the conventional generation fleet to meet the downward (during sunrise) and upward (during sunset) net load ramping needs. Another challenge corresponds to the ability of conventional generators to largely reduce their generation level or to turn off during a few hours in the middle of the day. If either of these two challenges cannot be dealt with by the generation fleet, solar power generation would need to be curtailed or the safe and reliable operation of the power system could be at risk. Accurate solar power forecasts would reduce the uncertainty of these challenges and would allow meeting minimum generation and ramping requirements in a more reliable and economic way through better unit commitment and economic dispatch decisions.

Unit commitment scheduling decisions vary significantly between power plants due to their different startup and shutdown times. Several power plant types must be committed one day ahead or earlier (e.g. nuclear, biomass, and coal). Other types of power plants instead can be recommitted a few hours ahead (e.g. gas combined cycle, as well as gas and oil steam turbines), while others may be re-committed one hour ahead or less (e.g. gas and oil fast turbines and internal combustion engines). If solar power is present in the system, solar power forecasts will inform unit commitment decisions, together with electrical demand forecasts. In a system that includes wind power, wind power forecasts will also be considered.

Economic dispatch decisions are impacted by medium-term and short-term solar power forecasting as a direct result of the unit commitment decisions of power plants with different startup times. Moreover, shorter-term solar power forecasts could allow a more economic and reliable dispatch by reducing the uncertainty of solar power generation in dispatch decisions that already consider other uncertainties (electric demand and wind power) as well as transmission (e.g. congestion), generation (e.g. ramping), and load (e.g. demand response) constraints.

A plethora of solar power forecasting technologies currently exist, including numerical weather prediction models (NWP), statistical models, and hybrid combinations of both [4]. The accuracy of solar power forecasts varies largely depending on the forecasting horizon, with errors generally decreasing as the horizon is reduced, e.g. one hour-ahead (HA) forecast errors are on average smaller than one day-ahead (DA) forecast errors. However, recent work has shown that prediction error is more dependent on geographical locale (i.e. influences on time series characteristics) than the machine learning routine utilized for forecasting [5]. Machine learning has been a major influence on the improvements associated with solar power forecasting in recent years. Parallel research has shown commonly used error metrics provide nearly indistinguishable information [6], but machine learning can increase the accuracy of solar power forecasts up to $30 \%$ over state-of-the-art NWP models [7]. It is expected this accuracy will be continually enhanced in the future, but it is unclear what impact it will have on grid operations. Intuition says solar power forecasting is beneficial for power system operations, especially for higher solar power penetrations, but what is the point at which returns diminish?

Tuohy et al. [8] provided an overview of solar forecasting, including equipment (e.g. sky and satellite imagery), forecasting approaches (e.g. time series analysis and NWP), and practical experiences with solar forecasting with respect to distributed and behind-the-meter experiences. Kaur et al. [9] showed how improvements in solar power forecasting reduced the probability of an imbalance caused through wrong market bids from PV solar plants, as well as flexibility 
reserve requirements. Nevertheless, the economic value of solar power forecasting improvement has not yet been fully examined in the literature. The lion's share of research at the interface of forecasting and the power grid has revolved around wind power. Early work by Milligan et al. examined the economics of an accurate wind forecast using measured utility data, and provided ranges of value estimates using production cost modeling [10]. Roulston et al. examined the consolidation of strategic information for improved generation decision making [11]. More recent wind power forecasting research has focused on market impacts [12], carbon constraints [13], market integration [14], island systems [15], pool-based electricity markets [16], and a short-term, technology-agnostic study for forecasting value quantification [17].

This article investigates the value of solar power forecasting improvements, both in terms of variable electricity generation costs and its impact on bulk power system operations. The study is performed by simulating the operation of the Independent System Operator - New England (ISO-NE) power system under a range of scenarios with varying solar power penetrations and solar power forecasting improvements. These scenarios are compared on a cost basis, including generation, fuel expenditures, variable operation and maintenance (VO\&M) costs, and start and shutdown costs. Moreover, operational impacts on conventional electricity generators are analyzed. These include total electricity generation and hourly ramping. The impacts on solar power curtailment are also investigated, showing how relatively large solar power penetrations are possible and cost effective from an operational standpoint.

Following this introduction section, the article is structured as follows. Section II describes the production cost model used within the study, as well as the scenarios under which the operation of the ISO-NE power was simulated. Section III presents and discusses the results as divided in two parts: section 3.1 analyzes the impact of increasing solar power penetration assuming stateof-the-art solar power forecasts, while section 3.2 analyzes the impact of uniform solar power forecasting improvements for various solar power penetration levels.

\section{Methodology}

The value of solar power forecasting improvement and its impact on bulk power system operations were analyzed by simulating the operation of the ISO-NE power system under several scenarios with varying solar power penetration levels, for a range of uniform solar power forecasting improvements.

A production cost model of the ISO-NE power system [18] was used to simulate its hourly operation for one year, including unit commitment and economic dispatch decisions with a mixed-integer programming (MIP) optimality gap of $0.1 \%$. The model was implemented in PLEXOS [19], a commercial production cost modeling tool, and simulated for DA and real-time (RT) markets considering transmission, generation, load, and reserve constraints.

Although many ISOs now operate sub-hourly energy markets, for the variables examined in this work, such as generation mixes and overall system costs, hourly RT markets produce very similar results to sub-hourly markets [20]. There are however variables, such as locational marginal prices (LMPs), where using a sub-hourly RT markets would produce significantly 
different results, and would be advisable. In addition, in this work we hold reserves, but do not actually model the deployment of the reserves, a common assumption in integration cost studies [21-23]. If reserve deployment were a focus of the study the modeling of the sub-hourly RT markets would be necessary. In addition, over a geographic area as large as the ISO-NE many of the sub-hourly variations in solar power are less pronounced due to the geographic smoothing caused by the aggregation of a large number of solar sites [24].

The model holds both contingency and regulation operating reserves. Electricity flows are simulated nodally and constrained for voltage levels equal to and above $69 \mathrm{kV}$. Generators are committed in the DA or RT runs depending on their start up times. For instance, nuclear, biomass, coal, hydro, gas combined-cycle (CC), and gas and oil steam turbine (ST) generators are committed in the (hourly) DA run; while gas and oil turbines (GT) and internal combustion (IC) generators are committed in the (hourly) RT run. Hydroelectric generation was also dispatched in the DA run. The ISO-NE electricity generation mixture, as represented in the model, includes the conventional generators (excluding solar power) present in ISO-NE in 2010. Details on the generation representation of the ISO-NE PLEXOS model can be found in a recent publication (see chapter 2.2 in [18]). The model takes into consideration several generation constraints and properties, including variable operation and maintenance costs (VO\&M), fuel costs (from Energy Information Administration (EIA) 2006 data), piecewise linear heat rate functions (heat rates, and plant efficiency accordingly, are a function of the load factor), upward and downward ramp rates, minimum up and down times, and start and shutdown costs. The values used here for the ISO-NE model correspond to real generator properties and constraints that are classified as critical energy infrastructure information (CEII) and therefore cannot be made publicly available.

Figure 1 shows the share of the different electricity generation technologies, amounting to a total installed capacity of $34.2 \mathrm{GW}$. Hydro pumping and electricity exchanges with neighboring regions were not considered in this modeling exercise in order to isolate and better understand the impacts of solar power and of solar power forecasting improvement on bulk power system operations.

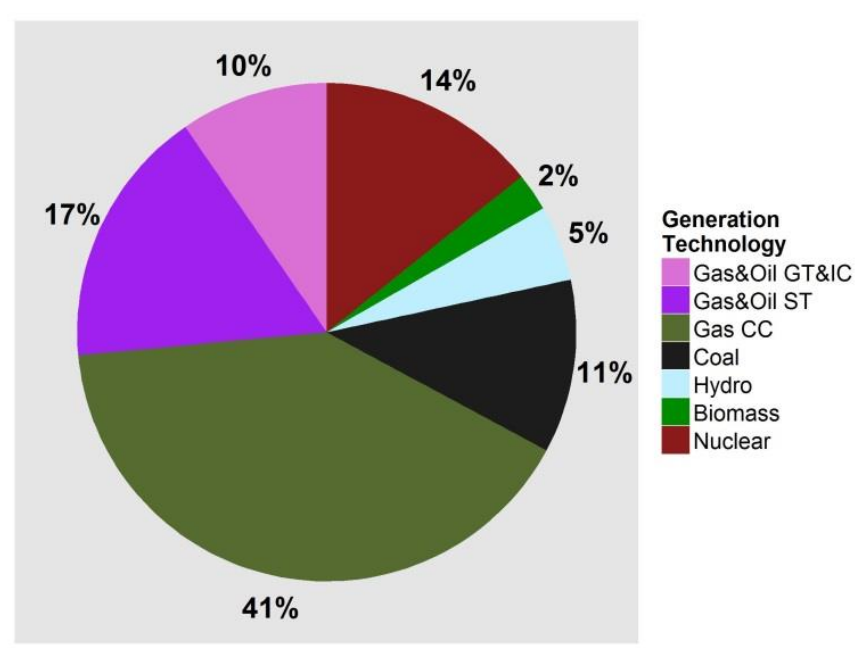

Figure 1 - Electricity Generation Mix 
NREL's solar power data for integration study [25] was the source for the solar power data used for the different solar penetration scenarios. The database includes 68 utility-scale solar power plants and 76 distributed solar power plants in ISO-NE. The total installed capacity of all the sites in the database present in ISO-NE amounts to $4,874 \mathrm{MW}$, equivalent to a $4.5 \%$ solar power penetration by energy. Figure 2 illustrates the solar power available from the 144 solar power plants included in the database with a carpet plot mapped by month and hour of the day.

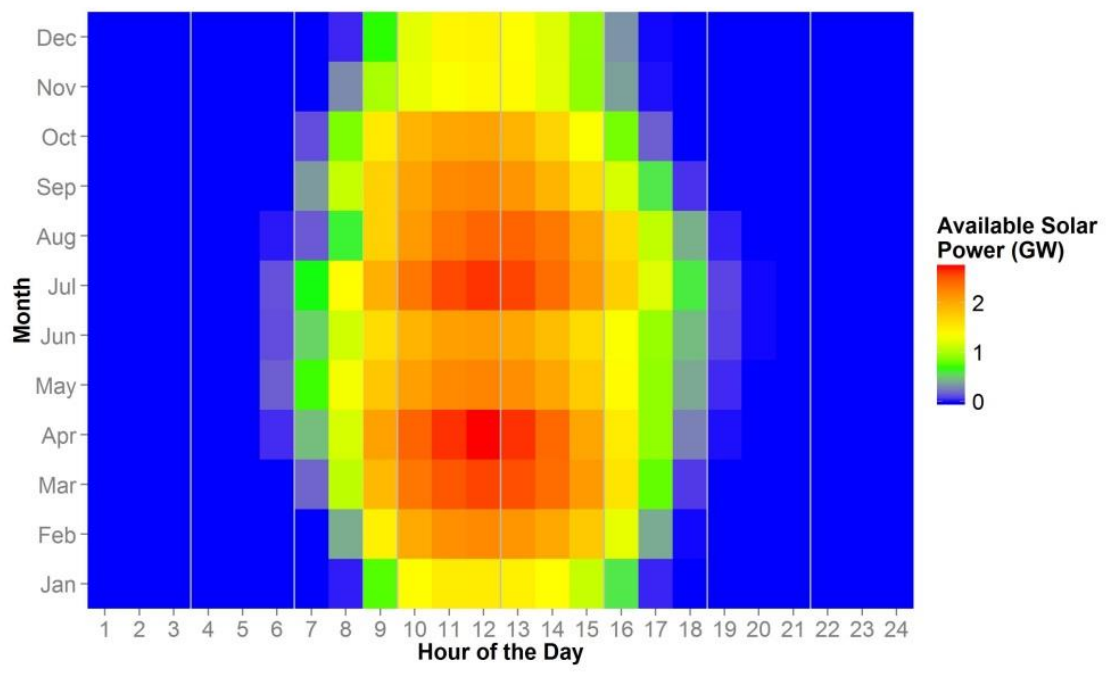

Figure 2 - Available Solar Power

In order to simulate scenarios with solar power penetration levels larger than $4.5 \%$, the solar power plant units were multiplied by 2,3 , and 4 to obtain respective solar power penetrations of $9 \%, 13.5 \%$, and $18 \%$. The utility-scale and the distributed solar power plants are well distributed throughout the ISO-NE power system; thus, the solar output smoothing for higher solar penetrations is the same as for the $4.5 \%$ solar penetration. An examination of the spatial smoothing for various subsets of the data revealed that there was very little additional smoothing when a large number of plants $(>20)$ were considered. When additional plants were considered beyond $4 \%$ penetration, the normalized hour-to-hour solar variability for the four scenarios with increasing solar power penetrations $(4.5 \%, 9 \%, 13.5 \%$, and $18 \%)$ is the same and equal $6.73 \%$. Solar power curtailment was only modeled for utility-scale solar power plants; it was assumed that distributed solar power plants (rooftop PV) could not be curtailed by the system operator.

To consider correlations between solar irradiance and electricity demand, the same weather year (2006) was used for the load [26] and solar data [25]. The DA load and solar power forecasts were included in the DA run in order to take into account forecasting errors in the unit commitment decision process. The DA load forecasts are included in the ISO-NE load database [26], while the DA solar power forecasts are derived from a state-of-the-art NWP model, namely the Weather Research and Forecasting Model (WRF) [27]. Details on the WRF setup to mimic operational solar irradiance day-ahead forecasts are available on the NREL website [25]. Figure 3 shows the distribution of the errors for the state-of-the-art DA solar power forecasts. In order to analyze the impact of solar power forecasting improvement, the DA solar power forecasts were uniformly improved for various levels up to perfect forecasting (i.e. $100 \%$ uniform improvement). 


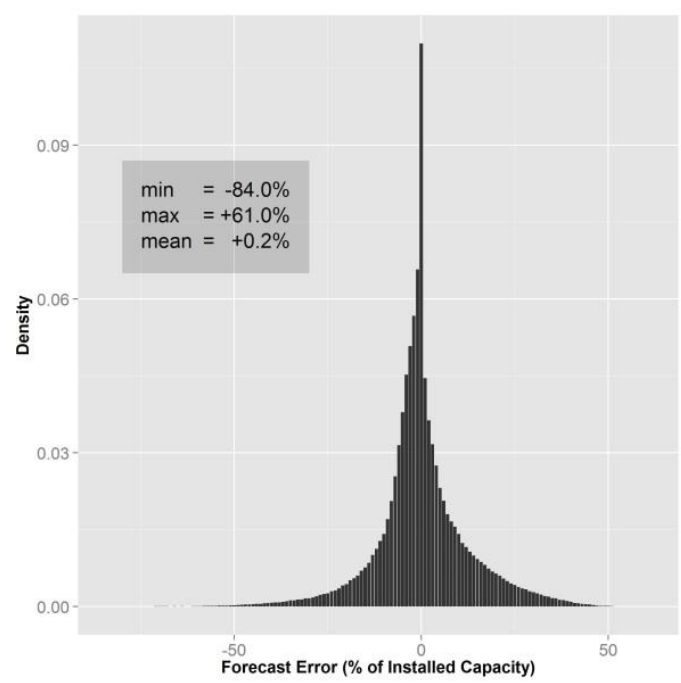

Figure 3 - Distribution of DA Solar Power Forecast Errors

The uniform solar forecast improvements were accomplished through hindcasting, i.e. an artificial improvement to historic forecasts. The approach was adopted to ascertain the value of solar forecasting for grid operations, the research focus herein, and because adjustments, modifications, and complementary models to standard NWP models can individually lead to superior or inferior solar forecasts based on sky conditions. For instance, the Aerosol-based Forecasts of Solar Irradiance for Energy Applications (AFSOL) model has shown superior accuracy to the European Centre for Medium-Range Weather Forecasts (ECMWF) model in clear sky conditions and inferior accuracy in cloudy conditions [28, 29]. Although there are an uncountable number of possible models and parameterizations, parallel research has shown model blending and machine learning can improve solar forecasting accuracy by over 30\% [30]. In this article, the assumption of uniform improvement was justified by the bulk energy perspective and the stated desire to assess first-order impacts of improved solar power forecasting. One year of hourly bulk power system operations of the ISO-NE power system was simulated for 21 scenarios: one without solar power, and 20 scenarios with varying solar power penetration levels $(4.5 \%, 9 \%, 13.5 \%$, and $18 \%)$ with state-of-the-art DA solar power forecasts, as well as for a range of uniform solar power forecasting improvements $(25 \%, 50 \%, 75 \%$, and $100 \%)$. 

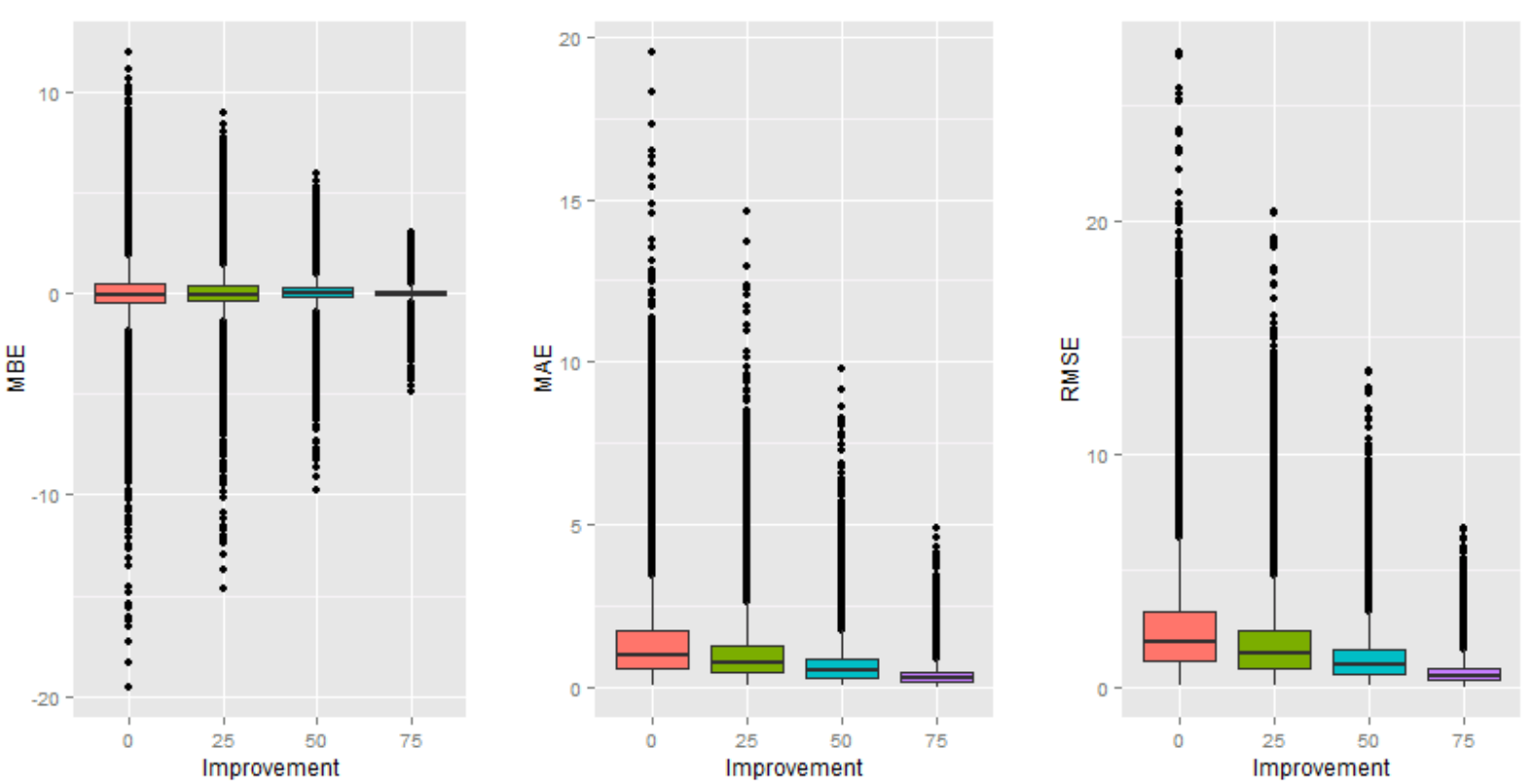

Figure 4 - Box plots of Day-Ahead Forecasting Errors as Quantified by MBE, MAE, and RMSE Metrics

Common metrics such as the mean bias error (MBE), mean absolute error (MAE), and root mean square error (RMSE) are useful for comparing solar power forecasts [6]. In this article the forecasts were produced to start and end at midnight. Metrics were thus calculated for every day of the year to compare day-ahead errors against each other and results from comparable studies. Figure 4 illustrates these metric distributions with box plots, and Table 1 provides percentile information. It is important to note that the statistics shown below are for the aggregated solar power forecasts over the entire study area. Due to geographic smoothing these metric values are likely much lower than one would see for individual sites.

Table 1 - Percentiles of MBE, MAE, and RMSE Metrics for Day-Ahead Forecasting Errors

\begin{tabular}{|c|c|c|c|c|c|c|c|c|c|c|c|c|}
\cline { 2 - 11 } \multicolumn{1}{c|}{} & \multicolumn{10}{c|}{} & \multicolumn{10}{c|}{ Improvement [\%] } & $\mathbf{0}$ & $\mathbf{1 0}$ & $\mathbf{2 0}$ & $\mathbf{3 0}$ & $\mathbf{4 0}$ & $\mathbf{5 0}$ & $\mathbf{6 0}$ & $\mathbf{7 0}$ & $\mathbf{8 0}$ & $\mathbf{9 0}$ & $\mathbf{1 0 0}$ \\
\hline \multirow{4}{*}{ MBE } & $\mathbf{0 . 0}$ & -19.51 & -1.09 & -0.58 & -0.34 & -0.19 & -0.06 & 0.11 & 0.34 & 0.70 & 1.34 & 11.91 \\
\cline { 2 - 11 } & $\mathbf{2 5 . 0}$ & -14.63 & -0.82 & -0.44 & -0.26 & -0.14 & -0.05 & 0.08 & 0.26 & 0.52 & 1.00 & 8.93 \\
\cline { 2 - 11 } & $\mathbf{5 0 . 0}$ & -9.75 & -0.54 & -0.29 & -0.17 & -0.10 & -0.03 & 0.05 & 0.17 & 0.35 & 0.67 & 5.95 \\
\hline \multirow{4}{*}{ MAE } & $\mathbf{7 5 . 0}$ & -4.88 & -0.27 & -0.15 & -0.09 & -0.05 & -0.02 & 0.03 & 0.09 & 0.17 & 0.33 & 2.98 \\
\cline { 2 - 11 } & $\mathbf{0 . 0}$ & 0.01 & 0.33 & 0.48 & 0.63 & 0.79 & 0.98 & 1.21 & 1.51 & 1.95 & 2.83 & 19.51 \\
\cline { 2 - 11 } & $\mathbf{2 5 . 0}$ & 0.01 & 0.25 & 0.36 & 0.47 & 0.59 & 0.73 & 0.91 & 1.13 & 1.47 & 2.12 & 14.63 \\
\cline { 2 - 11 } & $\mathbf{5 0 . 0}$ & 0.01 & 0.17 & 0.24 & 0.31 & 0.39 & 0.49 & 0.61 & 0.75 & 0.98 & 1.42 & 9.75 \\
\hline \multirow{4}{*}{ RMSE } & $\mathbf{7 5 . 0}$ & 0.00 & 0.08 & 0.12 & 0.16 & 0.20 & 0.24 & 0.30 & 0.38 & 0.49 & 0.71 & 4.88 \\
\cline { 2 - 11 } & $\mathbf{0 . 0}$ & 0.04 & 0.65 & 0.92 & 1.22 & 1.55 & 1.90 & 2.32 & 2.86 & 3.66 & 5.26 & 27.21 \\
\cline { 2 - 11 } & $\mathbf{2 5 . 0}$ & 0.03 & 0.49 & 0.69 & 0.92 & 1.16 & 1.43 & 1.74 & 2.15 & 2.74 & 3.95 & 20.41 \\
\cline { 2 - 11 } & $\mathbf{5 0 . 0}$ & 0.02 & 0.32 & 0.46 & 0.61 & 0.77 & 0.95 & 1.16 & 1.43 & 1.83 & 2.63 & 13.61 \\
\hline & $\mathbf{7 5 . 0}$ & 0.01 & 0.16 & 0.23 & 0.31 & 0.39 & 0.48 & 0.58 & 0.72 & 0.91 & 1.32 & 6.80 \\
\hline
\end{tabular}




\section{Results and Analysis}

The annual production cost modeling results of the 21 simulated scenarios were analyzed in terms of bulk power system operations, including operational electricity generation costs, electricity generation and ramping of conventional generators, and solar power curtailment. The results are structured and analyzed in two parts. Section 3.1 analyzes the impact of increasing solar power penetrations, while section 3.2 analyzes the impact and the value of increasingly improving DA solar power forecasting.

\subsection{Impact of Solar Power}

The impact of increasing solar power penetration was analyzed by simulating bulk power system operations in ISO-NE for five scenarios, one without solar power and four with increasing solar power penetration levels which include state-of-the-art DA solar power forecasts. Figure 5 shows the electricity generation mix for the five scenarios. The electricity generation sources most displaced by increasing solar power penetration were gas CC, as well as gas and oil ST (46\% and $32 \%$ respective reductions with $18 \%$ solar power penetration). Biomass and coal generators also decreased their share with increasing solar power $(6 \%$ and $7 \%$ respective reduction with $18 \%$ solar power penetration). The only electricity generation technology that increased its share as solar power penetration increased was gas and oil GT and IC. This electricity generation type corresponds to the largest ramping capabilities as well as the highest variable generation costs. The additional variability and uncertainty that solar power provides increase largely the use of faster generators. For instance, gas and oil GT and IC electricity generation increased by $188 \%$ with an $18 \%$ solar power penetration, from 0.91 to $2.62 \mathrm{TWh}$. Solar power curtailment also increased with higher solar power penetrations. No solar curtailment resulted from the scenario with $4.5 \%$ solar power penetration. With larger solar power penetrations of $9 \%, 13.5 \%$, and $18 \%$, solar power curtailment increased to $0.5 \%, 3.3 \%$, and $9.5 \%$ of available solar power respectively. The increase is not linear but rather exponential due to the higher curtailment need for the marginal solar generator as penetration increases. For the larger penetrations analyzed, curtailment was also partly caused by transmission congestion as the distribution of solar power is kept the same. 


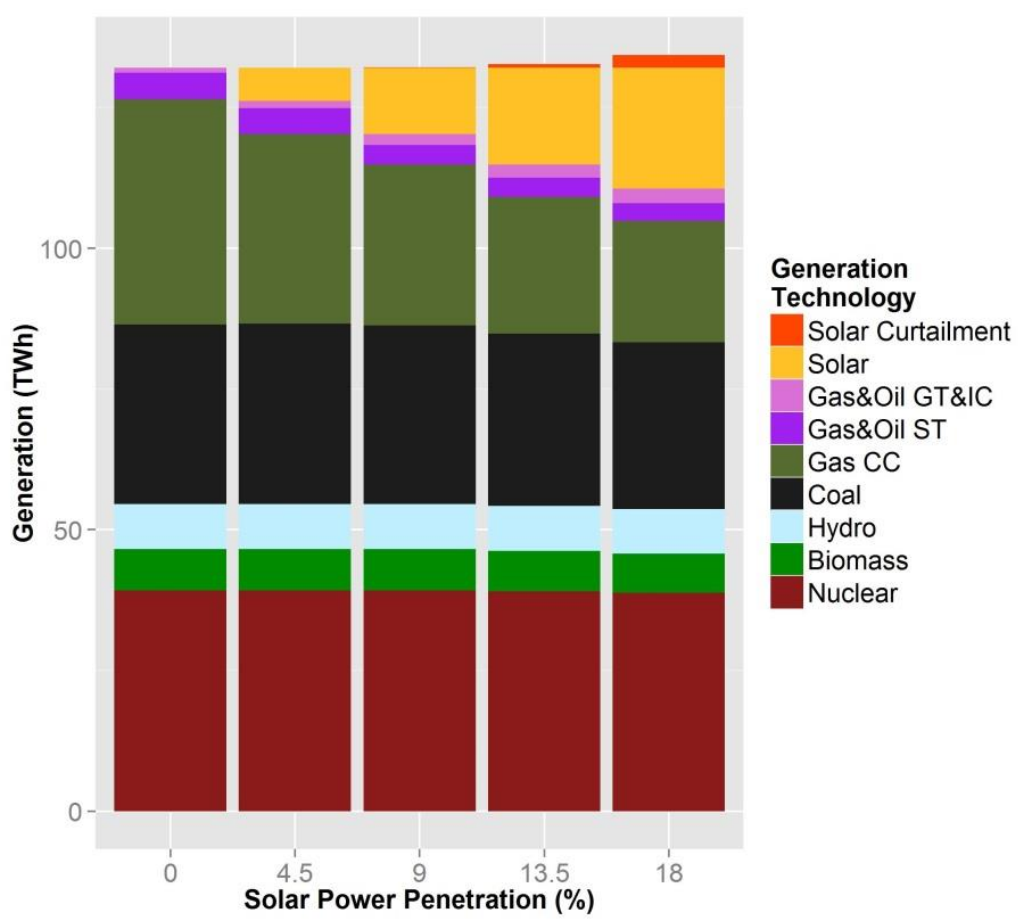

Figure 5 - Electricity Generation Mix for Different Solar Power Penetrations

The impact of solar power on other electricity generation sources does not only impact their share in the electricity generation mix, it also impacts the way in which they are operated. Figure 6 shows how the increasing penetration of solar power increased upward ramping of all electricity generation sources, even the ones whose generation mix share decreased. Upward ramping is defined as the annual sum of the magnitudes, in power terms, of each individual upward ramping event for each power plant belonging to a specific electricity generation source. For instance, gas $\mathrm{CC}$ almost halved its electricity generation with $18 \%$ solar power penetration while increasing upward ramping by 3\%. In other words, gas CC power plants generate less electricity with solar power penetration, but they are operated very differently, largely increasing their ramping relative to their generation level. A similar behavior was also observed for coal and for gas and oil ST generators. Nuclear especially, but also biomass generators are not designed to be ramped much nor often. The model included tight minimum generation level and ramping constraints for nuclear and biomass generators. With no solar power and with low solar power penetration levels, both nuclear and biomass electricity generation are constant. However, with larger penetrations of solar power both nuclear and biomass generators exercise ramping to a small extent within their operating constraints. A small amount of ramping of nuclear power plants was observed due to the minimum generation constraint being set to $90 \%$ of the installed capacity. This is not currently performed at many operational nuclear plants, but should be within the technical capabilities of most plants. Hydroelectric generation did not increase as solar power increased given its negligible generation cost, however the variability and uncertainty of solar power changed the way hydro was operated by increasing its ramping behavior. Gas and oil GT and IC power plants were also operated differently as solar power penetration increased. They ramped up and down more often as solar power increased. Upward ramping of gas and oil GT and IC was almost 4 times larger with $18 \%$ solar power penetration. 


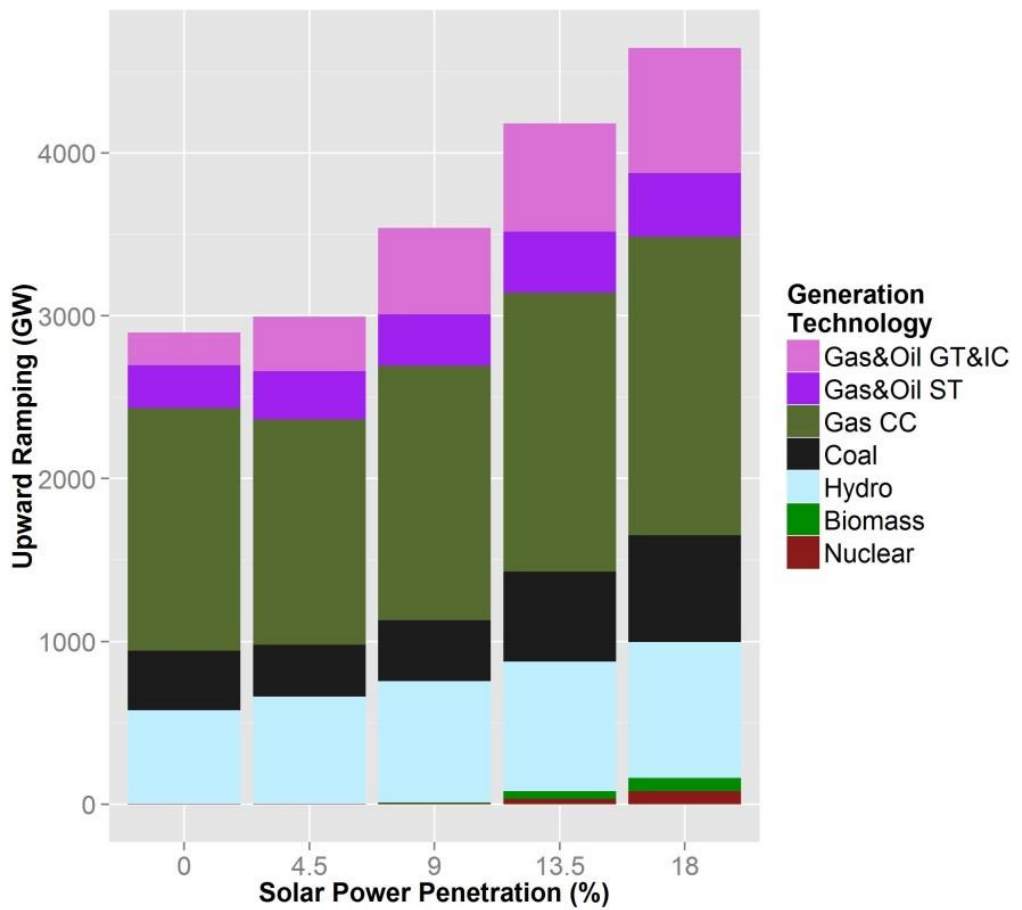

Figure 6 - Upward Ramping for Different Solar Power Penetrations

Solar power penetration also impacted the operation of some conventional generators in terms of their starts and shutdowns. As shown in Table 2, the variability and uncertainty of solar power largely increased the start and shutdown costs of gas CC, as well as gas and oil GT and IC generators. It is very interesting how the electricity generation share of gas CC almost halved while its start and shutdown costs more than doubled with $18 \%$ solar power penetration.

Table 2 - Start \& Shutdown Costs for Different Solar Power Penetrations

\begin{tabular}{|c|c|c|c|c|c|}
\hline Solar Power Penetration (\%) & 0.0 & 4.5 & 9.0 & 13.5 & 18.0 \\
\hline \hline Gas CC (M\$) & 38.7 & $\begin{array}{c}30.1 \\
(-22.1 \%)\end{array}$ & $\begin{array}{c}44.0 \\
(+13.7 \%)\end{array}$ & $\begin{array}{c}65.5 \\
(+69.3 \%)\end{array}$ & $\begin{array}{c}83.2 \\
(+115.1 \%)\end{array}$ \\
\hline Gas \& Oil GT \& IC (M\$) & 9.7 & $\begin{array}{c}15.9 \\
(+63.6 \%)\end{array}$ & $\begin{array}{c}29.7 \\
(+204.8 \%)\end{array}$ & $\begin{array}{c}38.0 \\
(+290.7 \%)\end{array}$ & $\begin{array}{c}45.7 \\
(+369.6 \%)\end{array}$ \\
\hline
\end{tabular}

The impact of solar power penetration on the share of start and shutdown costs for total operational generation costs is shown in Figure 7. Total operational generation costs include variable operation and maintenance costs (VO\&M), fuel costs, and start and shutdown costs. System-wide fuel and VO\&M generation costs decreased as solar power increased mainly due to the reduction in electricity generation from gas CC, gas and oil ST, coal, and biomass generators, as shown in Figure 5. For instance, the sum of fuel and VO\&M costs decreased by $16.5 \%$ and $27.2 \%$ with $9 \%$ and $18 \%$ solar power penetration respectively. The rate of decrease of fuel and VO\&M costs decreased for lower solar power penetrations due to the increase in ramping, start and shutdown costs, and solar power curtailment. 


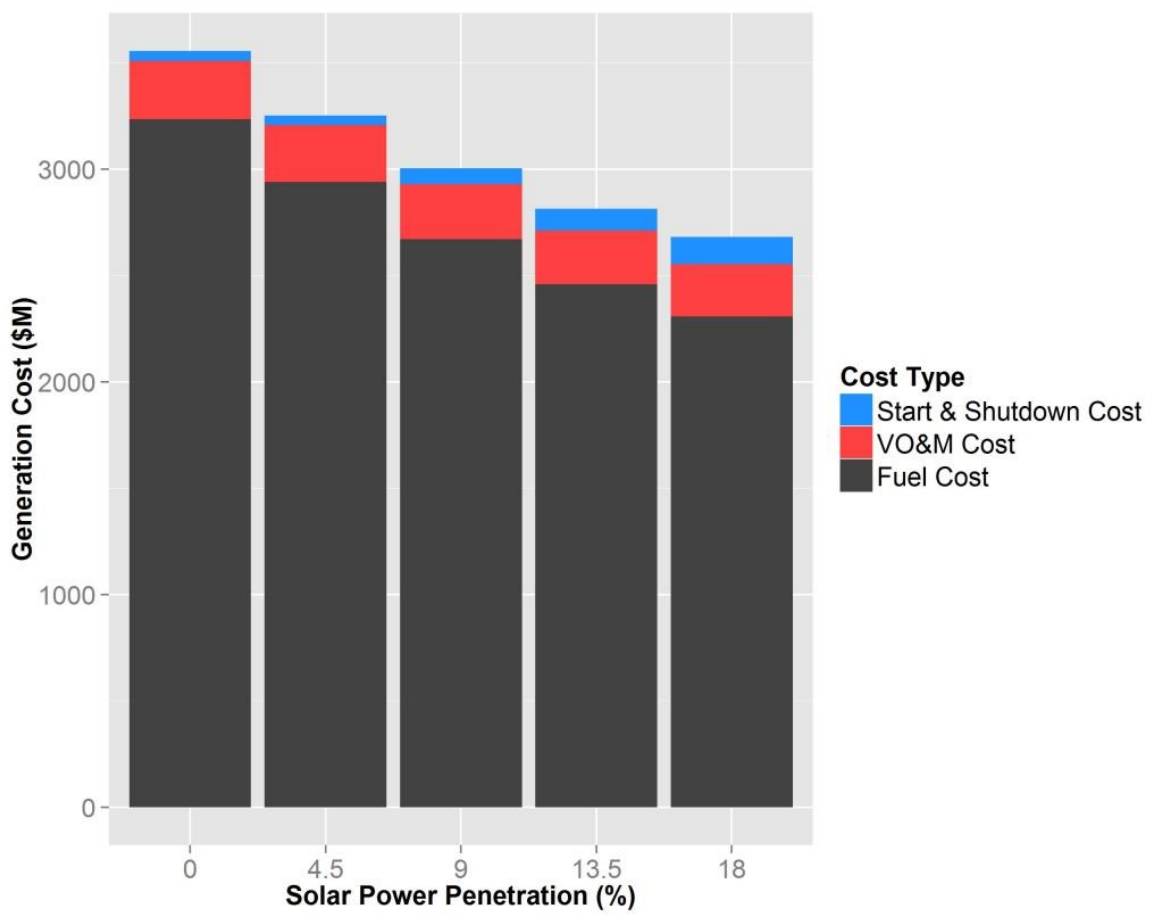

Figure 7 - Generation Costs per Cost Type for Different Solar Power Penetrations

$4 \quad$ System-wide start and shutdown costs increased with solar power mainly due to the increased 5 startups and shutdowns of gas CC, as well as of gas and oil GT and IC generators, as shown in 6 Table 2. For instance, start and shutdown costs increased by 52\% and $167 \%$ with $9 \%$ and $18 \%$ 7 solar power penetration respectively. The rate of increase of start and shutdown costs increased 8 as solar power penetration increased due to the increasing need to start and shutdown 9 conventional power plants to cope with the variability and uncertainty of solar power.

11 The resulting system-wide variable generation cost decreased by $15.5 \%$ and $24.9 \%$ with $9 \%$ and $1218 \%$ solar power penetration, respectively. The impacts of solar power on conventional 13 generators, their ramping as well as their start and shutdown behavior explained in this section 14 resulted in smaller variable generation costs for gas CC, coal, and gas and oil ST generators. 15 Operational generation costs instead increased for gas and oil GT and IC generators, as shown in 16 Figure 8. 


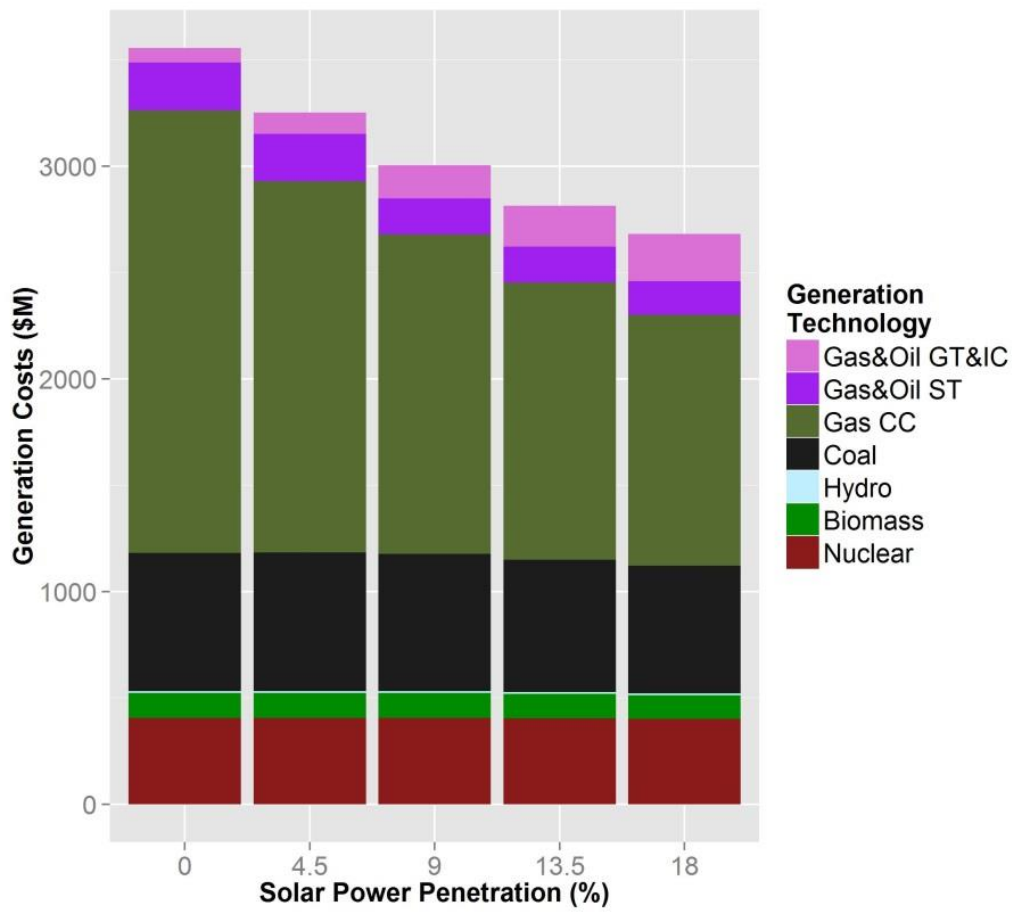

Figure 8 -Generation Costs per Generation Type for Different Solar Power Penetrations

The results presented in this section are useful to understand the impact of solar power on the operation of the ISO-NE bulk power system. As mentioned in the methodology section of this paper, the unit commitment and economic dispatch model used for the analysis did not consider electricity exchanges with neighboring regions nor hydro pumping. Moreover, hydro generation is also dispatched in the DA run without allowing it to be re-dispatched in the RT run. In real operations the arbitrage provided by electricity imports and exports, hydro pumping, and hydro generation re-dispatch would reduce the impacts of the variability and uncertainty of solar power estimated here.

\subsection{Impact of Solar Power Forecasting Improvement}

The impact of solar power forecasting improvement was analyzed by evaluating bulk power system operations for four scenarios with increasing solar power penetrations using state-of-theart DA solar power forecasts as well as four improved forecasts that represent four different uniform improvement levels: 25\%, 50\%, 75\%, and 100\% (perfect forecast). Table 3 shows how solar power forecasting improvements impact electricity generation of fossil fueled conventional generators for different solar power penetrations (numbers show differences compared to stateof-the-art forecasting). Electricity generation from more expensive and faster generation sources (gas and oil GT and IC generators) decreased with solar power forecasting improvement. In other words, faster generators committed in RT were used less as solar power uncertainty decreased. 
Table 3 - Impact of Solar Power Forecasting Improvement on Electricity Generation

\begin{tabular}{|c||c|c|c|c||c|c|c|c||c|c|c|c||c|c|c|c|}
\hline \multicolumn{1}{|c||}{ Solar Penetration (\%) } & \multicolumn{3}{|c||}{4.5} & \multicolumn{4}{|c||}{9.0} & \multicolumn{4}{|c||}{13.5} & \multicolumn{5}{c|}{18.0} \\
\hline Forecast Improvement (\%) & 25 & 50 & 75 & 100 & 25 & 50 & 75 & 100 & 25 & 50 & 75 & 100 & 25 & 50 & 75 & 100 \\
\hline \hline Coal (\% change) & 0.0 & 0.0 & 0.0 & 0.1 & 0.1 & 0.2 & 0.2 & 0.3 & 0.1 & 0.3 & 0.4 & 0.5 & 0.2 & 0.4 & 0.4 & 0.4 \\
\hline Gas CC (\% change) & 0.0 & 0.2 & 0.1 & 0.1 & 0.1 & 0.1 & 0.2 & 0.3 & 0.3 & 0.7 & 0.9 & 1.1 & 0.5 & 0.9 & 1.4 & 1.8 \\
\hline Gas \& Oil ST (\% change) & -0.1 & -1.4 & -0.8 & -0.8 & 0.6 & 1.2 & 0.5 & 0.0 & -1.3 & -2.8 & -3.9 & -4.8 & -1.9 & -3.5 & -5.3 & -5.5 \\
\hline Gas \& Oil GT \& IC (\% change) & -0.8 & -1.8 & -1.9 & -1.7 & -4.5 & -7.5 & -8.6 & -10.2 & -3.9 & -8.8 & -12.1 & -13.7 & -5.1 & -10.5 & -14.3 & -17.5 \\
\hline
\end{tabular}

2

Figure 9 shows the average difference in electricity generation from gas and oil GT and IC generators when DA solar power forecasting was uniformly improved by $50 \%$, per month and hour of the day, compared to the state-of-the-art forecasting case. Electricity generation from more expensive and faster generators decreased especially during sunset (refer to the solar resource in Figure 2). Reducing an over-forecasting error during sunset reduced the need to generate electricity from gas and oil GT and IC generators used to counteract the solar power forecast error during the evening electricity demand peak.

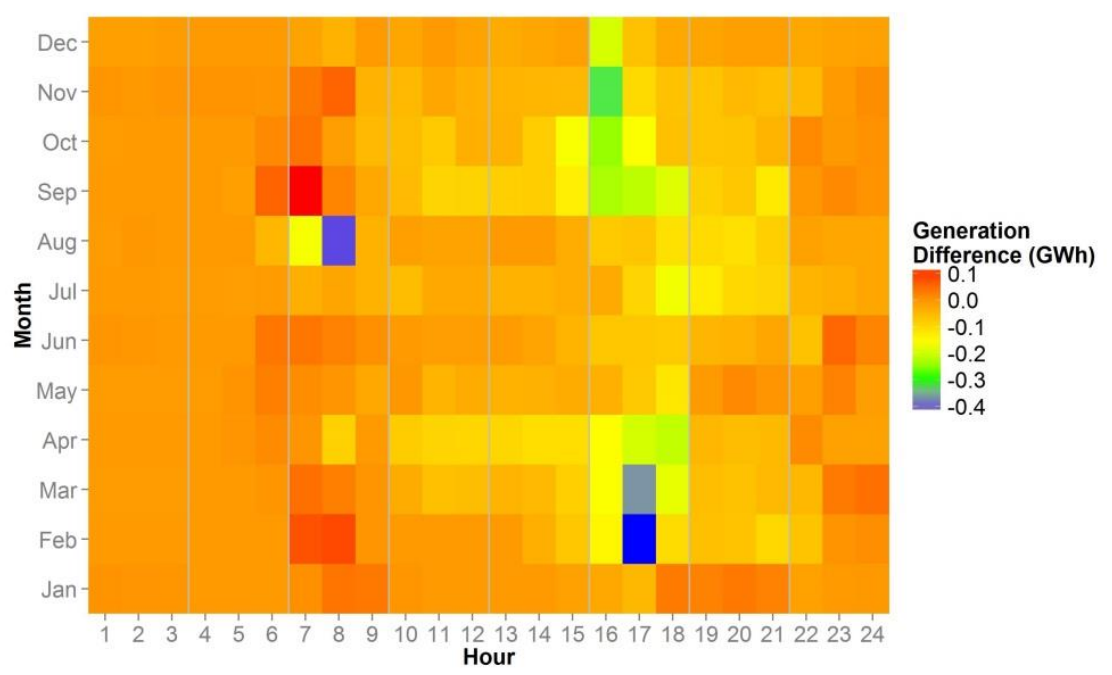

Figure 9 - Impact of 50\% Uniform Solar Power Forecasting Improvement on Gas \& Oil GT \& IC Electricity Generation

Table 3 also shows how electricity generation from another expensive source committed in the DA run (gas and oil ST generators) also decreased with improved solar power forecasting; this is especially true for the higher solar power penetrations. The decrease in electricity generation from the more expensive sources was balanced by an increase in electricity generation from cheaper sources (coal and gas CC generators) that were committed in the DA run. The need for ramping and re-dispatchment decreased as solar power uncertainty decreased. Table 3 not only shows how the impact of solar power forecasting improvement on electricity generation was larger for higher solar power penetrations, it also shows how the additional impacts of forecasting improvement decreased for additional uniform improvements. In other words, the impacts of improving state-of-the-art solar power forecasting by 50\% were larger than further improving forecasts from a 50\% uniform improvement level to perfect forecasts (100\% uniform improvement). 
The decrease in electricity generation from more expensive sources was also balanced by a decrease in solar power curtailment as shown in Table 4 (numbers show differences compared to state-of-the-art forecasting). Decreasing DA solar power uncertainty decreased the need to curtail solar power in RT. The absolute impact on solar power curtailment increased with solar power penetration. For example, a 50\% solar power forecasting uniform improvement reduced solar power curtailment by $2.2 \mathrm{GWh}$ and by $62.1 \mathrm{GWh}$ with $9 \%$ and $18 \%$ solar power penetration respectively. As shown in Table 4, the relative reduction of solar power curtailment compared to state-of-the-art forecasting did not increase with solar power penetration. The main reason for this is that for the highest solar power penetrations solar power curtailment was partly caused by transmission congestion and it was much larger, as shown in Figure 5.

Table 4 - Impact of Solar Power Forecasting Improvement on Solar Power Curtailment

\begin{tabular}{|c|c|c|c|}
\hline Impact (\% change) & \multicolumn{3}{|c|}{ Solar Penetration (\%) } \\
\hline Forecast Improvement (\%) & 9.0 & 13.5 & 18.0 \\
\hline \hline 25 & -2.3 & -1.2 & -1.5 \\
\hline 50 & -4.0 & -2.3 & -2.7 \\
\hline 75 & -5.2 & -3.5 & -3.5 \\
\hline 100 & -5.4 & -4.2 & -3.8 \\
\hline
\end{tabular}

The impact of solar power forecasting improvement on other electricity generation sources did not only impact their share in the electricity generation mix, it also impacted the way in which they operated. Table 5 shows how improving solar power forecasting reduced upward ramping of all fossil fueled electricity generators (numbers show differences compared to state-of-the-art forecasting). Ramping reduction lowered fuel costs because the operating points of power plants varied less in time and they are operated at more efficient load points. Ramping reductions increased with solar power penetration, as well as with solar power forecasting improvement level. As DA solar power uncertainty decreased power plants needed to ramp less in RT. Similarly to the case of fossil fueled electricity generation shown in Table 4, Table 5 shows how the impact of forecasting improvement on ramping decreased for additional uniform improvements for most cases. The largest impact on ramping of solar power forecasting improvement was observed for the two most expensive sources of electricity generation, gas and oil ST, as well as gas and oil GT and IC.

Table 5 - Impact of DA Solar Power Forecasting Improvement on Electricity Generation Upward Ramping

\begin{tabular}{|c||c|c|c|c||c|c|c|c||c|c|c|c||c|c|c|c|}
\hline \multicolumn{1}{|c||}{ Solar Penetration (\%) } & \multicolumn{3}{|c||}{4.5} & \multicolumn{3}{|c||}{9.0} & \multicolumn{3}{|c||}{13.5} & \multicolumn{3}{|c|}{18.0} \\
\hline Forecast Improvement (\%) & 25 & 50 & 75 & 100 & 25 & 50 & 75 & 100 & 25 & 50 & 75 & 100 & 25 & 50 & 75 & 100 \\
\hline \hline Coal (\% change) & -0.5 & -0.7 & -1.1 & -1.7 & -1.1 & -3.5 & -5.4 & -6.0 & -1.1 & -2.9 & -4.7 & -5.2 & -1.5 & -3.0 & -4.0 & -4.5 \\
\hline Gas CC (\% change) & -0.5 & -0.7 & -1.5 & -1.7 & -0.2 & -1.2 & -2.1 & -2.4 & -0.4 & -1.0 & -1.8 & -1.9 & -0.3 & -0.8 & -1.6 & -1.5 \\
\hline Gas \& Oil ST (\% change) & -0.9 & -3.2 & -2.6 & -2.8 & -2.4 & -3.3 & -5.7 & -7.7 & -3.9 & -8.5 & -11.7 & -14.2 & -3.1 & -7.7 & -11.8 & -16.8 \\
\hline Gas \& Oil GT \& IC(\% change) & -0.2 & -0.9 & -1.1 & -1.0 & -2.4 & -3.5 & -3.5 & -4.3 & -1.6 & -3.5 & -4.6 & -5.7 & -2.9 & -5.3 & -6.9 & -9.5 \\
\hline
\end{tabular}

Solar power forecasting improvements also impacted the operation of some conventional generators in terms of their startups and shutdowns. As shown in Table 6, the reduction of DA solar power uncertainty reduced the start and shutdown costs of gas and oil GT and IC generators. These expensive and fast reacting generators were needed less with improved solar 
power forecasting due to the reduction in net load (load minus solar power) uncertainty. Table 3 also shows how these power plants generated less electricity with improved DA solar power forecasts. In contrast, startup and shutdown costs of gas CC generators increased with improved solar power forecasting. Table 3 also shows how gas CC power plants generated more electricity with improved DA solar power forecasts.

Table 6 - Impact of Solar Power Forecasting Improvement on Start \& Shutdown Costs

\begin{tabular}{|c||c|c|c|c||c|c|c|c||c|c|c|c||c|c|c|c|}
\hline \multicolumn{1}{|c||}{ Solar Penetration (\%) } & \multicolumn{4}{|c||}{4.5} & \multicolumn{4}{|c||}{9.0} & \multicolumn{4}{|c||}{13.5} & \multicolumn{5}{|c|}{18.0} \\
\hline Forecast Improvement (\%) & 25 & 50 & 75 & 100 & 25 & 50 & 75 & 100 & 25 & 50 & 75 & 100 & 25 & 50 & 75 & 100 \\
\hline \hline $\begin{array}{c}\text { Gas CC } \\
\text { \% change) }\end{array}$ & 0.3 & 1.1 & 1.7 & 3.2 & 1.6 & 2.3 & 3.8 & 5.5 & 0.0 & 0.9 & 2.4 & 4.3 & 0.4 & 0.1 & 1.8 & 2.9 \\
\hline $\begin{array}{c}\text { Gas \& Oil GT \& IC } \\
\text { (\% change) }\end{array}$ & -0.1 & -1.0 & -1.0 & -1.4 & -3.5 & -4.9 & -5.3 & -6.2 & -1.5 & -3.5 & -5.1 & -6.3 & -2.7 & -4.9 & -7.0 & -9.9 \\
\hline
\end{tabular}

The impact of solar power forecasting improvements on solar power curtailment as well as on electricity generation, ramping, and starts and shutdowns of fossil fueled electricity generators resulted in impacts on operational electricity generation costs (sum of fuel costs, VO\&M costs, and start and shutdown costs). Figure 10 shows the economic value of uniform solar power forecasting improvements for different solar power penetrations, in terms of the reduction in annual operational electricity generation costs. The value of solar power forecasting improvement was larger for higher solar power penetrations. The normalized value per unit of solar power generation increased with solar power penetration, as also shown in Table 7. For example, a $25 \%$ uniform solar power forecasting improvement reduced annual operational electricity generation costs by $\$ 3.92 \mathrm{M}$ (equivalent to an average of $\$ 0.33$ savings per $\mathrm{MWh}$ of solar power generation) and by $\$ 10.65 \mathrm{M}$ (equivalent to an average of $\$ 0.50$ savings per MWh of solar power generation) for $9 \%$ and $18 \%$ solar power penetration, respectively. Figure 10 also shows how the additional value of solar power forecasting improvement decreased for additional uniform improvements. For example, with a $13.5 \%$ solar power penetration, uniformly improving DA state-of-the-art solar power forecasting by $50 \%$ reduced annual operational electricity generation costs by $\$ 13.22 \mathrm{M}$, while the additional value of improving solar power forecasting to $100 \%$ was only $\$ 6.34 \mathrm{M}$. The decreasing marginal value of solar forecasting improvements is explained by the fact that system operations were designed to handle variability and uncertainty in load. Even in these simulations, removing all solar power uncertainty still leaves load uncertainty that can lead to sub-optimal scheduling.

The value of solar power forecasting improvement is driven by several uncertainties associated with modeling assumptions and constraints. One of the main uncertainties that affect the modeling results presented in this paper in terms of forecasting improvement cost savings are fuel prices. The magnitude of the cost savings from solar power forecasting improvement are a function of the assumed fuel prices and the differences between them. 


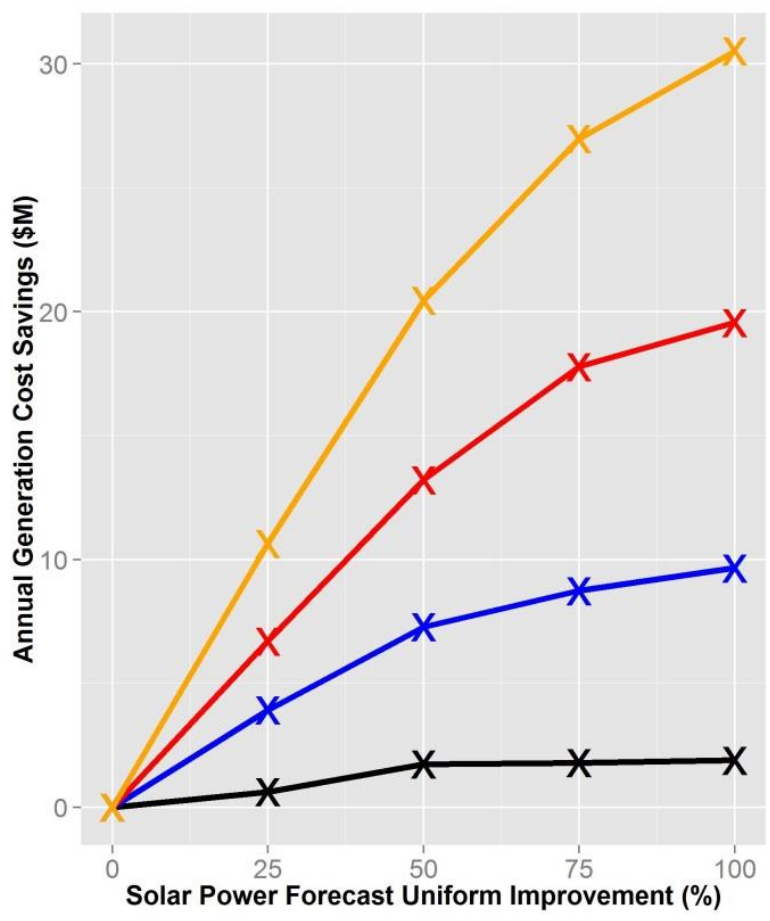

Solar Power

Penetration Level

$\times 18$

* 13.5

* 9

× 4.5

Figure 10 - Variable Electricity Generation Cost Savings from Solar Power Forecasting Improvement

Table 7- Cost Savings from Solar Power Forecasting Improvement per Unit of Solar Power Generation

\begin{tabular}{|c|c|c|c|c|}
\hline Forecasting Improvement Cost Savings (\$/MWh) & \multicolumn{5}{|c|}{ Solar Penetration (\%) } \\
\hline Forecast Improvement (\%) & 4.5 & 9.0 & 13.5 & 18.0 \\
\hline \hline 25 & 0.11 & 0.33 & 0.39 & 0.50 \\
\hline 50 & 0.29 & 0.62 & 0.77 & 0.95 \\
\hline 75 & 0.30 & 0.74 & 1.03 & 1.25 \\
\hline 100 & 0.32 & 0.82 & 1.13 & 1.42 \\
\hline
\end{tabular}

In order to better understand the value of DA solar power forecasting improvements and their impact on bulk power system operations, Figure 11 and Figure 12 show the electricity generation dispatch stack for an under-forecasting and an over-forecasting event respectively. Each figure shows the dispatch stack for one day for the DA (top row) and RT (bottom row) markets assuming state-of-the-art solar power forecasting (left column), as well as for $25 \%$ (center column) and 50\% (right column) uniform solar power forecasting improvements.

Figure 11 shows an example of how the power system operated following a large DA underforecast error, as well as how it would have potentially operated if the DA solar power forecast was uniformly improved by $25 \%$ and $50 \%$. The large DA under-forecast error caused solar power curtailment due to the over-commitment of conventional generators whose downward ramping capability was not large enough to allow the power system to absorb all the unexpected solar power generation. If DA solar power forecasting would have been improved, the resulting under-forecast error would have been smaller leading to the commitment of less gas CC generators during the middle of the day in the DA run. The smaller DA committed capacity 
would have therefore reduced the need to curtail solar power in RT. In this example, most of the value of solar power forecasting improvement came from the reduction in solar power curtailment and the resulting reduction in fossil fueled electricity generation, mainly from gas CC generators.
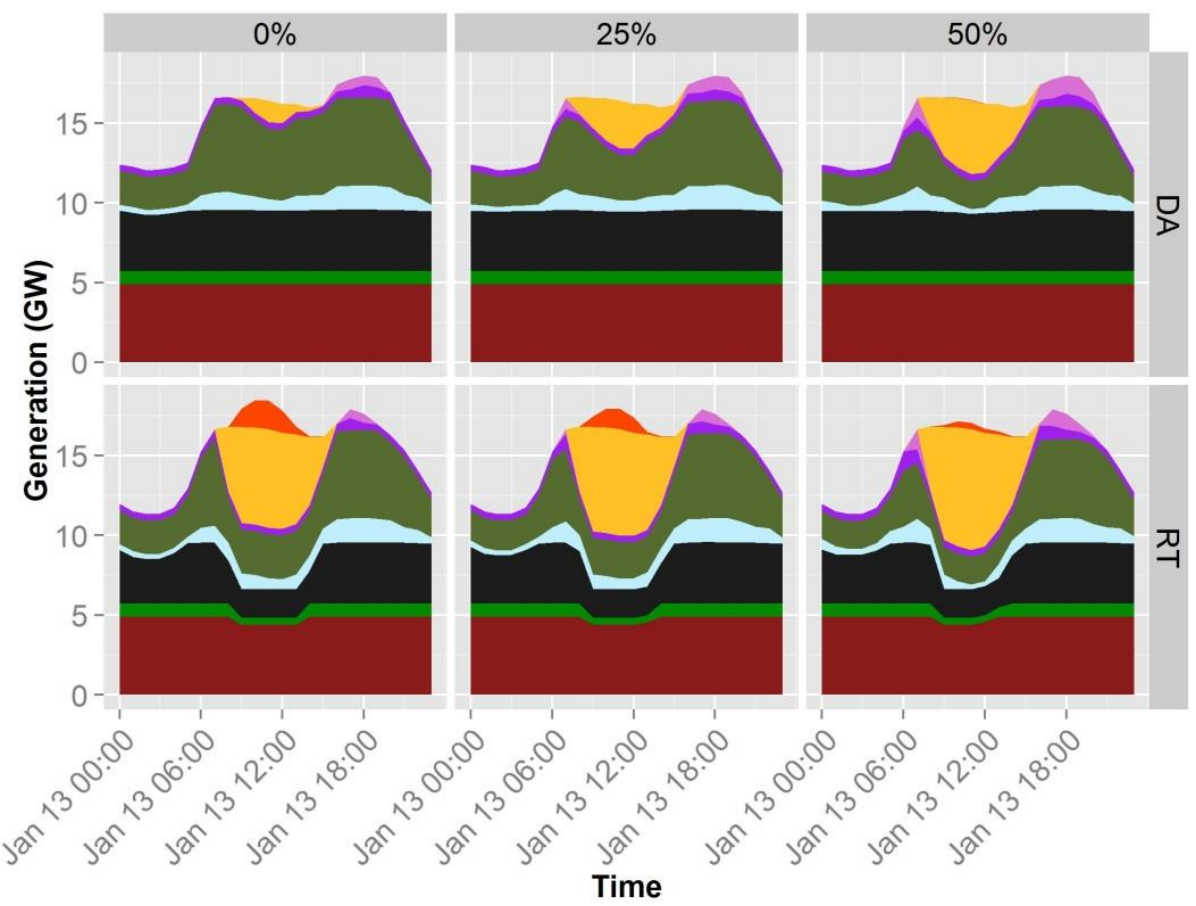

\author{
Generation \\ Technology \\ Solar Curtailment \\ Solar \\ Gas\&Oil GT\&IC \\ Gas\&Oil ST \\ Gas CC \\ Hydro \\ Coal \\ Biomass \\ Nuclear
}

Figure 11 - Solar Power Under-Forecasting Event

Figure 12 shows an example of how the power system operated following a large DA overforecast error, as well as how it would have potentially operated if the DA solar power forecast was uniformly improved by $25 \%$ and $50 \%$. The large DA over-forecast error caused fossil fueled generators committed in the DA run, such as coal, gas CC, and gas and oil ST generators, to ramp up their generation as much as their ramping constraints allowed them to. Moreover, in order to be able to meet RT electricity demand when solar power generation is way lower than what was forecasted in the DA run, gas and oil GT and IC generators are turned on and ramped up. Running gas and oil GT and IC generators is the most expensive form of generating electricity. If DA solar power forecasting would have been improved, the resulting over-forecast error would have been smaller leading to the commitment of more gas $\mathrm{CC}$ generators during the middle of the day in the DA run. The larger DA committed capacity would have therefore reduced the need to ramp up electricity generators committed in the DA run and especially the need to turn on the expensive and fast-reacting gas and oil GT and IC generators. In this example, most of the value of solar power forecasting improvement came from reducing the need to start up electricity generators with high fuel costs and from reducing the ramping need from fossil fueled generators committed in the DA run. 


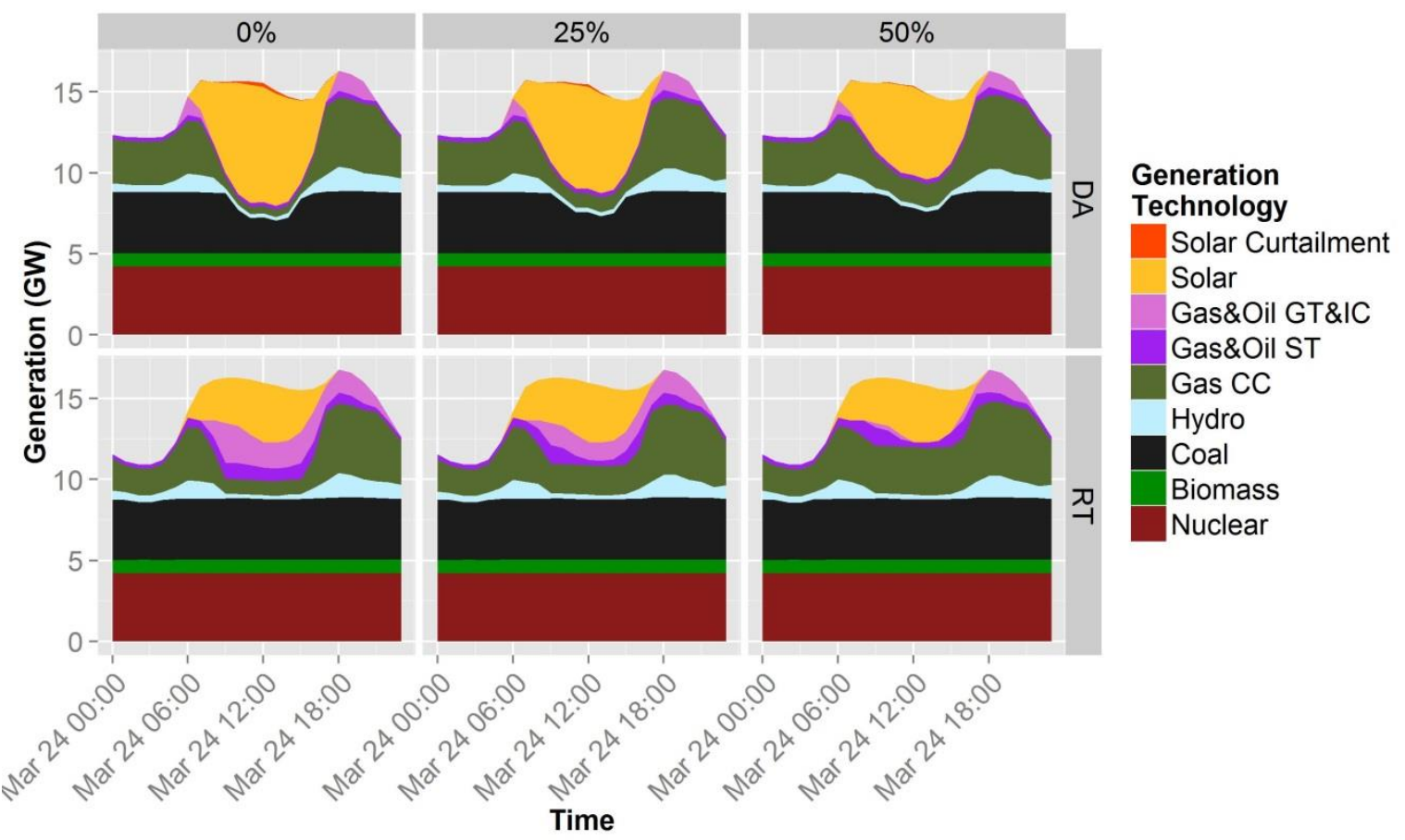

Figure 12 - Solar Power Over-Forecasting Event

The results presented in this section are useful to understand the value of solar power forecasting improvement and its impact on the operation of the ISO-NE bulk power system. Even if the unit commitment and economic dispatch model used for the analysis did not consider electricity exchanges with neighboring regions nor hydro pumping, and hydro generation was dispatched in the DA run without allowing it to be re-dispatched in the RT run, the analysis of the results provides useful insights on the value of DA solar power forecasting improvement as well as its impact on bulk power system operations. In real operations the arbitrage provided by electricity imports and exports, hydro pumping, and hydro generation re-dispatch, as well as a potential intra-day market, could partially reduce the value of solar power forecasting improvement. Nonetheless, the discussion of the results presented in this paper helps to understand and evaluate the need for solar power forecasting improvements in a power system with large penetrations of solar power with limited temporal and spatial arbitrage options, or in other words, with potential flexibility challenges raised by the variable and uncertain nature of solar power.

Solar power is a source of uncertainty in a power system. Solar power forecasting errors are a measure of such uncertainty. Power systems are designed to deal with uncertainties. The ability of the power system to arbitrage partly corresponds to their ability to reliably react to an unexpected event. Larger solar power forecast errors increase the need for arbitrage in a power system, and at the same time they increase the value of arbitrage options. For example, the value of and need for arbitrage options such a transmission, energy storage, demand response, and faster ramping plants increase as solar power uncertainty (or in other words, solar power forecasting errors) increases in the power system. 


\section{Conclusions}

The discussion of the results presented in this paper lead to interesting insights regarding the value of solar power forecasting improvement in terms of operational electricity generation costs as well as its impact on bulk power system operations. Several scenarios in terms of solar power penetration and uniform DA solar power forecasting improvement were evaluated using a production cost model of the ISO-NE power system.

In order to better understand the value of solar power forecasting improvement, the impact of solar power on bulk power system operations was analyzed assuming state-of-the-art solar power forecasting. The integration of variable and uncertain solar power decreased annual operational electricity generation costs, by decreasing fuel and VO\&M costs while decreasing start and shutdown costs of fossil fueled conventional generators. The only conventional electricity sources that increased their share in the generation mix with increasing solar power penetration were gas and oil GT and IC generators due to their fast reacting capabilities and their ability to start within an hour during the hourly RT market simulation. Ramping of all fossil fueled generators and solar power curtailment also increased with the integration of increasing amounts of solar power.

The results analyzed throughout the paper show how solar power forecasting improvements changed the impacts that the uncertainty of solar power has on bulk power system operations. Electricity generation from the fast start and lower efficiency power plants, such as gas and oil GT and IC, was reduced when solar power forecasts were improved. Ramping of all generators, start and shutdown costs, and solar power curtailment also decreased with solar power forecasting improvements. All these impacts led to a reduction in overall operational electricity generation costs in the system that translates into an annual economic value of improving solar power forecasting. For instance, one of the results shows how improving DA state-of-the-art solar power forecasts in ISO-NE with a $13.5 \%$ solar power penetration decreased annual variable electricity generation costs by $\$ 13.2 \mathrm{M}$. This analysis only took into account the value of solar power forecasting from the production cost point of view at the day-ahead timescale. Improving solar forecasting at other timescales could also bring value to the system, in conjunction with, or independent of, day-ahead forecasts. There are also additional value streams from improving solar power forecasts that are not accounted for in the modeling, such as improved maintenance scheduling and reduced reserve levels. The results show how the marginal value of solar power forecasting improvement increased with solar power penetration, while it decreased with additional improvement levels.

Future work could continue to develop the analysis presented in this paper by analyzing the impact of including and improving shorter-term, intra-day and/or hour-ahead, solar power forecasting. Another interesting way forward could be to understand how the value of solar power forecasting presented in this paper would change with the integration of different flexibility options such as cross-border transmission, energy storage, demand response, and faster ramping generators. 


\section{Acknowledgments}

This work was supported by the U.S. Department of Energy under Contract no. DE-AC36-08GO28308 with the National Renewable Energy Laboratory as part of the project work performed under the SunShot Initiative's Improving the Accuracy of Solar Forecasting program. The authors would like to thank the entire Watt-Sun Project Team, in particular Jon Black of ISONE.

\section{References}

1. Candelise, C., M. Winskel, and R.J.K. Gross, The dynamics of solar PV costs and prices as a challenge for technology forecasting. Renewable and Sustainable Energy Reviews, 2013. 26: p. 96-107.

2. Barbose, G., et al., Costs and benefits of renewables portfolio standards in the United States. Renewable and Sustainable Energy Reviews, 2015. 52: p. 523-533.

3. Porter, K. and J. Rogers, Survey of variable generation forecasting in the west. Contract, 2012. 303: p. 275-3000.

4. Inman, R.H., H.T.C. Pedro, and C.F.M. Coimbra, Solar forecasting methods for renewable energy integration Progress in Energy and Combustion Science 2013. 39(6): p. 535 - 576.

5. Voyant, C., et al., Statistical parameters as a means to a priori assess the accuracy of solar forecasting models. Energy, 2015. 90, Part 1: p. 671-679.

6. Zhang, J., et al., A suite of metrics for assessing the performance of solar power forecasting. Solar Energy, 2015. 111: p. 157-175.

7. Zhang, J., et al., Baseline and Target Values for Regional and Point PV Power Forecasts: Toward Improved Solar Forecasting. Solar Energy, 2015(122): p. 804-819.

8. Tuohy, A., et al., Solar Forecasting: Methods, Challenges, and Performance. Power and Energy Magazine, IEEE, 2015. 13(6): p. 50-59.

9. Kaur, A., et al., Benefits of solar forecasting for energy imbalance markets. Renewable Energy, 2016. 86: p. 819-830.

10. Milligan, M.R., A.H. Miller, and F. Chapman, Estimating the economic value of wind forecasting to utilities. 1995: National Renewable Energy Laboratory Golden, CO.

11. Roulston, M.S., et al., Using medium-range weather forcasts to improve the value of wind energy production. Renewable Energy, 2003. 28(4): p. 585-602.

12. Jonsson, T., P. Pinson, and H. Madsen, On the market impact of wind energy forecasts. Energy Economics, 2010. 32(2): p. 313-320.

13. Higgins, P., et al., Impact of offshore wind power forecast error in a carbon constraint electricity market. Energy, 2014. 76: p. 187-197.

14. González-Aparicio, I. and A. Zucker, Impact of wind power uncertainty forecasting on the market integration of wind energy in Spain. Applied Energy, 2015. 159: p. 334-349.

15. Swinand, G.P. and A. O'Mahoney, Estimating the impact of wind generation and wind forecast errors on energy prices and costs in Ireland. Renewable Energy, 2015. 75: p. 468-473.

16. Mc Garrigle, E.V. and P.G. Leahy, Quantifying the value of improved wind energy forecasts in a pool-based electricity market. Renewable Energy, 2015. 80: p. 517-524.

17. Hodge, B., et al., The Value of Improved Short-Term Wind Power Forecasting. 2015, National Renewable Energy Laboratory (NREL), Golden, CO. 
18. Brancucci Martínez-Anido, C. and B. Hodge, Impact of Utility-Scale Distributed Wind on

Transmission-Level System Operations. 2014.

19. PLEXOS Integrated Energy Model. 2015; Available from:

http://energyexemplar.com/software/plexos-desktop-edition/.

20. Brady Stoll, G.B., Aaron Townsend, and Aaron Bloom, Analysis of Modeling Assumptions used in Production Cost Models for Renewable Integration Studies. 2015.

21. Lew, D. and R. Piwko, Western wind and solar integration study. National Renewable Energy Laboratories, Technical Report No. NREL/SR-550-47781, 2010.

22. D. Lew, G.B., E. Ibanez, A. Florita, M. Heaney, B.-M. Hodge, M. Hummon, G. Stark, J. King, S.A. Lefton, N. Kumar, D. Agan, G. Jordan, S. Venkataraman, The Western Wind and Solar Integration Study Phase 2. 2013.

23. GE, PJM Renewable Integration Study. 2012.

24. Mills, A., Implications of wide-area geographic diversity for short-term variability of solar power. Lawrence Berkeley National Laboratory, 2010.

25. NREL Solar Power Data for Integration Studies. Available from: http://www.nrel.gov/electricity/transmission/solar integration methodology.html.

26. ISO-NE load data. Available from: http://www.iso-ne.com/isoexpress/web/reports/pricing/tree/zone-info.

27. Skamarock, W.C., et al., A Description of the Advanced Research WRF Version 2, in NCAR TECHNICAL NOTE. 2005, NCAR: Mesoscale and Microscale Meteorology Division.

28. Wittmann, M., et al., Case Studies on the Use of Solar Irradiance Forecast for Optimized Operation Strategies of Solar Thermal Power Plants. IEEE Journal of Selected Topics in Applied Earth Observations and Remote Sensing, 2008. 1(1): p. 18-27.

29. Breitkreuz, H., et al., Short-Range Direct and Diffuse Irradiance Forecasts for Solar Energy Applications Based on Aerosol Chemical Transport and Numerical Weather Modeling. Journal of Applied Meteorology and Climatology, 2009. 48(9): p. 1766-1779.

30. Lu, S., et al., Machine Learning Based Multi-Physical-Model Blending for Enhancing Renewable Energy Forecast - Improvement via Situation Dependent Error Correction, in European Control Conference. 2015: Linz, Austria. 